\title{
Medical Fusion Components for a Web Dedicated Application
}

\author{
Ligia D. Chiorean ${ }^{1}$, Loreta A. Suta ${ }^{1}$, Mircea F. Vaida ${ }^{1}$, Mihaela Hedesiu ${ }^{2}$ \\ ${ }^{1}$ Department of Communications, Technical University of Cluj-Napoca, \\ Cluj-Napoca, Romania, \\ Chiorean.Ligia@com.utcluj.ro,Loreta.Suta@com.utcluj.ro, Mircea.Vaida@com.utcluj.ro. \\ ${ }^{2}$ Department of Oral Radiology, University of Medicine and Pharmacy Iuliu Hatieganu, \\ Cluj-Napoca, Romania, \\ mhedesiu@gmail.com.
}

\begin{abstract}
A web dedicated application is very useful for assisted diagnosis in healthcare domain. Medical images acquired with different medical image modalities contain important diagnostic information. The combination of complementary data from different images can lead to more important information. The fusion process allows combination of salient feature of these images. In this paper we present methods for medical image fusion, based on discrete wavelet transform. These methods are implemented in a web distributed application, using Java technology. The web dedicated application will integrate components able to be discovered in a ubiquitous computing system. The components are developed in dedicated Java packages that allow physicians to realize a fusion from a remote place. Considering radiology images we approve the functionality of the application by calculating significant quantitative parameters for fusion process evaluation.
\end{abstract}

Keywords: image fusion, wavelet transform, distributed application, multi-resolution decomposition

\section{Introduction}

In recent years, medical imaging became very useful for assisted diagnosis process. There are many medical image modalities that give important information about different diseases. These equipments are accompanied by software applications which offer image processing facilities. Many of these modalities offer complementary information. For example, CT (Computer Tomography) provides best information about denser tissue and MRI (Magnetic Resonance Image) offers better information on soft tissue, [4]. These complementarities have led to the idea that by combining images acquired with different medical devices can be obtained a new image that can offer more useful information. In this way, the result image can be very useful in the diagnosis process, and that is the main motif why image fusion has become an important research field.

The fusion techniques can be classified in two main categories: for spatial domain and for transform domain. The reason of passing to the transform domain is the fact that salience characteristics of the image are observed more easier than in spatial domain, and this is important to recognize informational underlayer of the image for fusing them comparing with the independent „combination” of the pixels. Fusion based on transforms has some advantages over other simple methods, like: energy compaction, larger SNR (Signal-to-Noise Ratio), reduced features, etc. The transform coefficients are representative for image pixels.

In a few past years, researchers developed different medical applications including image fusion. All these applications offer local solutions for the image fusion.

We propose a web architecture based on a web server for medical purposes. Remote fusion facilities offer many advantages in managing images obtained with specific medical devices in different locations. The implemented application features different fusion techniques to allow medical image processing in order to facilitate a better and faster diagnosis. We chose wavelet based fusion methods, namely: DWT (Discrete Wavelet Transform), UDWT (UnDecimated Wavelet Transform) and SIDWT (Shift Invariant Discrete Wavelet Transform). These techniques have been structured in software packages that allow future development depending on the clients' configuration (desktop, mobile, etc.) devices.

\section{Background}

Fusion can be realized on different abstraction levels: 
- Fusion on data level: data combination is realized directly on the image data. To realize such an operation the images have to be acquired by homogenous sensors.

- Fusion on feature level: using features extracted from images.

- Fusion on decision level: decisions are combined to the fusion result. Inhomogeneous sensors may be used for such a fusion, [2].

Some image fusion methods have been introduced in the literature, including statistical method (Bayesian's decision), Fuzzy set method, neural network method, Laplacian pyramid method and wavelet transform method, [4]. An effective fusion algorithm should integrate all the relevant information without generating additional artifacts.

DWT was the first transform used for image fusion in transform domain. For its application facilities and the quality of the obtained results this method is widely used. SIDWT is another wavelet transform used by Rockinger [10] in image fusion to eliminate the problems generated by shift variance of DWT.

The basic steps of the fusion technique using a multi-resolution decomposition are the following: the images are decomposed using a transform; a fusion rule is applied between the correspondent images obtained after the analysis step and an inverse transform is used for the synthesis step. The result of the inverse transform represents the fused image.

\section{Discrete Wavelet Transform in Image Fusion Process}

In this section we describe the basic principles of the image fusion in the transform domain for the following methods: DWT, UDWT and SIDWT.

The most transforms used in image proccesings split the image in important local components and transform methods become very important. Considering transform operator $\mathfrak{I}(\cdot)$ and fusion rule $g(\cdot)$, the fusion technique may be expressed as:

$$
I_{f}(x, y)=\mathfrak{J}^{-1}\left\{g\left(\mathfrak{J}\left\{I_{1}(x, y)\right\}, \ldots, \mathfrak{J}\left\{I_{T}(x, y)\right\}\right)\right\}
$$

The operator $g(\cdot)$ describes the information included from many input images. This operation represents the fusion rule.

\section{General discrete wavelet transform}

Wavelets are used for time frequency localization, and perform multi-scale and multi-resolution operations. Discrete wavelet transform (DWT) transforms a discrete time signal to a discrete wavelet representation. It converts an input series $x_{0}, x_{1}, \ldots, x_{m}$, into one high-pass wavelet coefficient series and one low-pass wavelet coefficient series (of length $\mathrm{n} / 2$ each) given by the (2) formulas, [1].

In practice, such a transformation will be applied recursively on the low-pass series until the desired number of iterations is reached.

$$
\begin{aligned}
& H_{i}=\sum_{m=0}^{k-1} x_{2 i-m} \cdot s_{m}(z) \\
& L_{i}=\sum_{m=0}^{k-1} x_{2 i-m} \cdot t_{m}(z)
\end{aligned}
$$

where $\mathrm{s}_{\mathrm{m}}(\mathrm{z})$ and $\mathrm{t}_{\mathrm{m}}(\mathrm{z})$ are called wavelet filters, $\mathrm{k}$ is the length of the filter, and $i=0,1, \ldots, \frac{n}{2}-1$.

Image fusion may be implemented by twodimensional discrete wavelet transform.

The DWT analyses the image at different frequency bands with different resolutions by decomposing the image into coarse approximation (LL) and detail coefficients (HL, LH and HH).

The transform is applied on the previously registered images. This operation generates coefficients for images. A fusion rule has to be established and applied on these coefficients. The fused image is obtained using inverse transform.

There are different fusion schemes that are implemented using DWT, such as:

- maximum selection (MS): chooses the coefficients with the maximum magnitude from every subband, [6].

- choose-max (CMax): computes the average value between the coefficients of the low-pass subband and selects the maximum value from high-pass correspondent subbands. 
$f^{(K)}(n \mid 0)=\frac{1}{P} \sum_{i=1}^{P} u_{i}^{(K)}(n \mid 0)$

$f^{(k)}(n \mid p)=\arg \max \left(\left|u_{i}^{(k)}(n \mid g)\right|\right)$

$\mathrm{k}=1 \ldots \mathrm{K}-1, \mathrm{~g}=1 \ldots \mathrm{G}$.

- weighted average (WA): This is a scheme developed by Burt and Kolczynski, [3] and is based on a normalized correlation between the two images sub-bands over a small local area. This measure is involved in computing a coefficient that is used in the fusion process.

- window based verification (WBV) scheme: This scheme creates a binary decision map to choose between each pair of coefficients using a majority filter [5].

\section{Undecimated discrete wavelet transform}

The translation invariance property is essential in image processing. In order to preserve the translation invariance property, some authors have introduced the concept of stationary wavelet transform. The downsampling operation is suppressed, but filters are upsampling by $2^{\mathrm{j}}$, for example by inserting $2^{\mathrm{j}}-1$ zeroes between any couple of consecutive coefficients.
In image fusion process, the source images are decomposed using UDWT. Two sets of wavelet coefficients are obtained, including approximation (LL) and detail (HL, LH and $\mathrm{HH}$ ) signals of the original data. A decision strategy establishes the rule for injecting the spatial details.

Aiazzi et al., [1] used this method for fusing 2 images with different resolutions. They used as fusion strategy a method based on the local correlation coefficients (LCC). These coefficients may be computed from the approximation coefficients (LL) of the source images, over a sliding square window.

Figure 1 shows the fusion procedure in the UDWT case for fusing 2 images acquired with different medical modalities. As fusion strategy we used a method proposed by Burt and Kolczynski, based on LCC. For comparison we have also implemented choose-max fusion rule.

The fusion method proposed by Burt and Kolczynski introduces two new measures: match - which determines the similarity degree of 2 image zones and salience - which represents the pertinent information (variance or energy) [3].

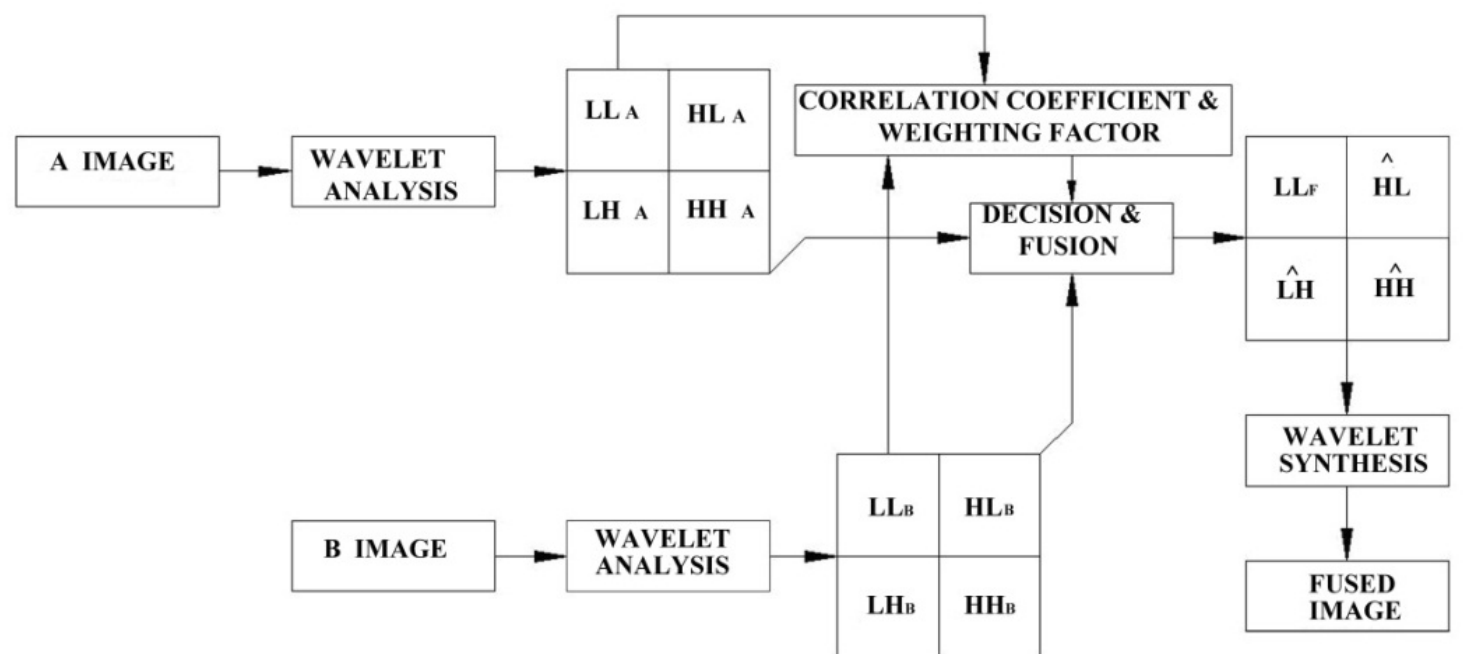

Figure 1. Undecimated wavelet-based image fusion procedure

UDWT (undecimated discrete wavelet transform) achieved exact shift invariance by discarding the downsampling and upsampling operations. However, the UDWT method is much more expensive in computation and storage due to the high degree of redundancy. 


$$
\begin{gathered}
s_{i}^{(k)}(n \mid g)=\sum_{\Delta n \in W^{(k)}}\left|u_{i}^{(k)}(n+\Delta n \mid g)\right|^{2} \\
m_{A B}^{(k)}(n \mid g)=\frac{2 \sum_{\Delta n \in W^{(k)}} u_{A}^{(k)}(n+\Delta n \mid g) \cdot u_{B}^{(k)}(n+\Delta n \mid g)}{s_{A}^{(k)}(n \mid g)+s_{B}^{(k)}(n \mid g)} \\
f^{(k)}(n \mid g)=\alpha_{A}(n \mid g) \cdot u_{A}^{(k)}(n \mid g)+ \\
+\left[1-\alpha_{A}(n \mid g)\right] \cdot u_{B}^{(k)}(n \mid g) \\
\alpha_{A}(n \mid g)=\left\{\begin{array}{l}
1 \\
0 \\
\frac{1}{2}+\frac{1}{2} \cdot\left(\frac{1-m_{A B}^{(k)}(n \mid g)}{1-\tau}\right) \\
\frac{1}{2}-\frac{1}{2} \cdot\left(\frac{1-m_{A B}^{(k)}(n \mid g)}{1-\tau}\right)
\end{array}\right) \\
\text { for }\left\{\begin{array} { l } 
{ m _ { A B } ^ { ( k ) } ( n | g ) \leq \tau } \\
{ m _ { A B } ^ { ( k ) } ( n | g ) > \tau } \\
{ m _ { A B } ^ { ( k ) } ( n | g ) > \tau }
\end{array} , \text { and } \left\{\begin{array}{l}
s_{A}^{(k)}(n \mid g) \leq \tau \\
s_{A}^{(k)}(n \mid g)>s_{B}^{(k)}(n \mid g) \\
s_{A}^{(k)}(n \mid g) \leq s_{B}^{(k)}(n \mid g)
\end{array}\right.\right.
\end{gathered}
$$

where:

- $\mathrm{S}_{\mathrm{i}}$ - energy of image $\mathrm{i}$ at level $\mathrm{k}$

- $\mathrm{m}_{\mathrm{AB}}$ - the matching measure (correlation)

- $\tau$ - the threshold

$-\alpha$ - the weighted factor

- $\mathrm{f}$ - the fusion rule

The third formula of relations (4) is applied for the corresponding pixels in the details images.

\section{Shift invariant discrete wavelet transform algorithm}

The discrete approach of the wavelet transform can be done with the special version of SIDWT, the so-called ATWT (à trous algorithm - with holes).

The "à trous" wavelet transform is a nonorthogonal multiresolution decomposition defined by a filter bank $\left\{h_{i}\right\}$ and $\left\{g_{i}=\delta_{i}-h_{i}\right\}$ with the Kronecker operator $\delta_{i}$ denoting an all-pass filter. The goal of this transform is to eliminate the sampling step and, as a result, the interpolation at the synthesis step.
In contrast with the pyramidal wavelet decomposition (used by DWT), in the "à trous" algorithm the number of pixels is the same for all wavelet planes and it is the same as in the original image. One of the interesting properties of the ATWT is that it is translation invariant. The wavelet planes resulting from the ATWT of a translated image are just a translation of the wavelet coefficients, [7].

At each level of the decomposition the general SIDWT separates the image into two sequences: the wavelet sequence $\left(\mathrm{w}_{\mathrm{i}}\right)$ which is preserved and the scale sequence $\left(\mathrm{s}_{\mathrm{i}}\right)$ which consists the input image for the next decomposition step.

$\left\{\begin{array}{l}w_{i+1}(n)=\sum_{k} g\left(2^{i} \cdot k\right) s_{i}(n-k) \\ s_{i+1}(n)=\sum_{k} h\left(2^{i} \cdot k\right) s_{i}(n-k)\end{array}\right.$

where $s_{0}$ is the original image, $g\left(2^{i} k\right)$ and $\mathrm{h}\left(2^{\mathrm{i}} \mathrm{k}\right)$ are the filters obtained at each level i from their prototypes $\mathrm{g}(\mathrm{k})$ and $\mathrm{h}(\mathrm{k})$.

The prototype low-pass filter is usually zerophase symmetric. For a J-level decomposition, the "á trous" wavelet accommodates a number of coefficients $\mathrm{J}+1$ time greater than the number of pixels. The two filters are applied as in the DWT case, on lines and on columns. At a new decomposition level the filters are redesigned by inserting 0 between the coefficients obtained at the inferior levels. As a result the multi-resolution loses its sense and the analysis becomes a multi-scale one.

Due to the absence of the decimation step, the synthesis is simply obtained by summing the detail levels to the approximations:

$$
A_{0}(m, n)=A_{J}(m, n)+\sum_{j=0}^{J-1} W_{j}(m, n)
$$

The fusion scheme is obtained by means of ATWT decomposition. For the practical implementation of the "á trous" algorithm a two-dimensional filter associated to the scaling function is used. In this paper, we use a scaling function that has a B3 cubic spline profile. This function leads to the following low-pass filter, defined as a convolution kernel, $h_{i}(j, k)$ : 


$$
\left(\begin{array}{ccccc}
\frac{1}{256} & \frac{1}{64} & \frac{3}{128} & \frac{1}{64} & \frac{1}{256} \\
\frac{1}{64} & \frac{1}{16} & \frac{3}{32} & \frac{1}{64} & \frac{1}{64} \\
\frac{3}{128} & \frac{3}{32} & \frac{9}{64} & \frac{3}{32} & \frac{3}{128} \\
\frac{1}{64} & \frac{1}{16} & \frac{3}{32} & \frac{1}{16} & \frac{1}{64} \\
\frac{1}{256} & \frac{1}{64} & \frac{3}{128} & \frac{1}{64} & \frac{1}{256}
\end{array}\right)
$$

The mirror filter is used for implementing the boundary condition.

\section{Fusion quality evaluation methods}

Often, quality assessment is carried out by human visual inspection. However, an objective approach is mostly desired where the knowledge of ground-truth is not assumed in order to perform the best fusion method for the given inputs.

To evaluate the performance of a fusion technique, different parameters may be considered: PSNR (Peak Signal to Noise Ratio), RMSE (Root Mean Square Error), SNR (Signal-to-Noise Ratio), or others based on the information theory. In this document we implemented two criteria: the first one based on the mutual information and the second one based on local saliencies.

The mutual information criterion requires one couple of parameters which can evaluate the fusion performance: fusion factor $(\mathrm{FF})$ and fusion symmetry (FS). Mutual information is the amount of information that one image contains about another. Considering 2 input images $\mathrm{A}, \mathrm{B}$ and a fused image $\mathrm{F}$, we can calculate the amount of information that $\mathrm{F}$ contains about $\mathrm{A}$ and $\mathrm{B}$, according to the relations, [12]:

$$
\begin{aligned}
& M_{F A}(f, a)=\sum_{f, a} p_{F A}(f, a) \cdot \log \frac{p_{F A}(f, a)}{p_{F}(f) \cdot p_{A}(a)} \\
& M_{F B}(f, b)=\sum_{f, b} p_{F B}(f, b) \cdot \log \frac{p_{F B}(f, b)}{p_{F}(f) \cdot p_{B}(b)}
\end{aligned}
$$

where:

- $p_{A}(a), p_{B}(a)$ and $p_{F}(f)$ are the probability density functions of the individual images, [11].

- $p_{F A}(f, a)$ and $p_{F B}(f, b)$ are the joint probability density functions
- $\mathrm{p}_{\mathrm{AB}}(\mathrm{a}, \mathrm{b})$ is the joint distribution

- $h(a, b)$ is the joint histogram

The image fusion performance measure can be defined as:

$$
M I_{F}^{A B}=M I_{F A}(f, a)+M I_{F B}(f, b)
$$

Relation (9) reflects the total amount of mutual information that the fused image $\mathrm{F}$ contains about $\mathrm{A}$ and $\mathrm{B}$. This measure represents the Fusion Factor FF, $\mathrm{FF}=\mathrm{MI}_{\mathrm{F}}^{\mathrm{AB}}$. The bigger the FF, the better the fusion process performs.

Another calculated measure is Fusion Symmetry (FS), which denotes the symmetry of the fusion process related to two input images.

$$
F S=a b s\left(\frac{M I_{F A}(f, a)}{M I_{F A}(f, a)+M I_{F B}(f, b)}-0.5\right)
$$

A small value for this parameter indicates a good quality for the fusion process.

Wank and Bovik have introduced an objective image quality metric based on fullreference image quality assessment, [13].

Considering two images $\mathrm{x}$ and $\mathrm{y}$ the Wang and Bovik image quality index can be defined as:

$Q_{0}=\frac{4 \cdot \sigma_{x y} \cdot \bar{x} \cdot \bar{y}}{\left(\sigma_{x}^{2}+\sigma_{y}^{2}\right) \cdot\left(\bar{x}^{2}+\bar{y}^{2}\right)}$

where:

$\sigma_{x y}$ is the covariance of the image, $\sigma_{x} \sigma_{y}$ are the variance (saliency) of an image within a window of $8 \times 8$ pixels and $\bar{x}, \bar{y}$ are the mean of $\mathrm{x}$ respectively the mean of $\mathrm{y}$.

The value $\mathrm{Q}_{0}$ is a measure for the similarity of the vectors $\mathrm{x}$ and $\mathrm{y}$ and takes values between -1 and 1 .

The local weight $\lambda(\mathrm{w})$ indicates the relative importance of image a compared to image $b$

$$
\lambda(w)=\frac{s(a \mid w)}{s(a \mid w)+s(b \mid w)}
$$

where $s(a \mid w)$ and $s(b \mid w)$ are the two local saliencies of the two input images $a$ and $b$.

Piella et al., [8] propose novel quality factors for fusion, considering the Wang and Bovik image quality index. One of them is quality index $Q(a, b, f)$ that can be defined as: 


$$
\begin{aligned}
Q(a, b, f) & =\frac{1}{|W|} \sum_{w \in W}\left(\lambda(w) \cdot Q_{0}(a, f \mid w)+\right. \\
& \left.+(1-\lambda(w)) \cdot Q_{0}(b, f \mid w)\right)
\end{aligned}
$$

where $\mathrm{a}$ and $\mathrm{b}$ are the two input images and $\mathrm{f}$ is the fused image, $\mathrm{W}$ is the family of all windows and $|\mathrm{W}|$ is the cardinality of $\mathrm{W}$.

The overall saliency of a window is:

$$
C(w)=\max (s(a \mid w), s(b \mid w))
$$

The weighted fusion quality index $\mathrm{Q}_{\mathrm{w}}(\mathrm{a}, \mathrm{b}, \mathrm{f})$ is a variant of fusion quality index by giving more weight to those windows where the saliency of the input images is higher. These are likely to be perceptually important parts of the underlying scene.

$$
\begin{gathered}
Q_{W}(a, b, f)=\frac{1}{|W|} \sum_{w \in W} c(w) \cdot\left(\lambda(w) \cdot Q_{0}(a, f \mid w)+\right. \\
\left.+(1-\lambda(w)) \cdot Q_{0}(b, f \mid w)\right)
\end{gathered}
$$

where

$$
c(w)=C(w) / \sum_{w^{\prime} \in W} C\left(w^{\prime}\right)
$$

These factors do not require a reference image, so they may offer objective evaluation about the fusion quality. These factors were computed for each of the implemented fusion methods, in order to compare their performances.

\section{System Description}

The main objective of this paper is to develop a dedicated web application that will be used in healthcare for assisted diagnosis.

The important features of the proposed software application would be:

- the remote data access and manipulation;

- the on-line image processing facilities.

The application is intended to realize the management of the patients and their information, including images associated with medical investigations. It offers the possibility to add patients and images, to search patients by name, numeric identification or by diagnosis.

Software modules regarding image processing are provided, one of these offering image fusion facilities.

The described fusion methods are implemented in Java packages that may be considered as software components, integrated in the web distributed application, (Figure 2). These facilities may be useful for physicians who need image processing tasks from locations that do not provide such capabilities. The application runs on the server and the results are presented to the user in a web browser.

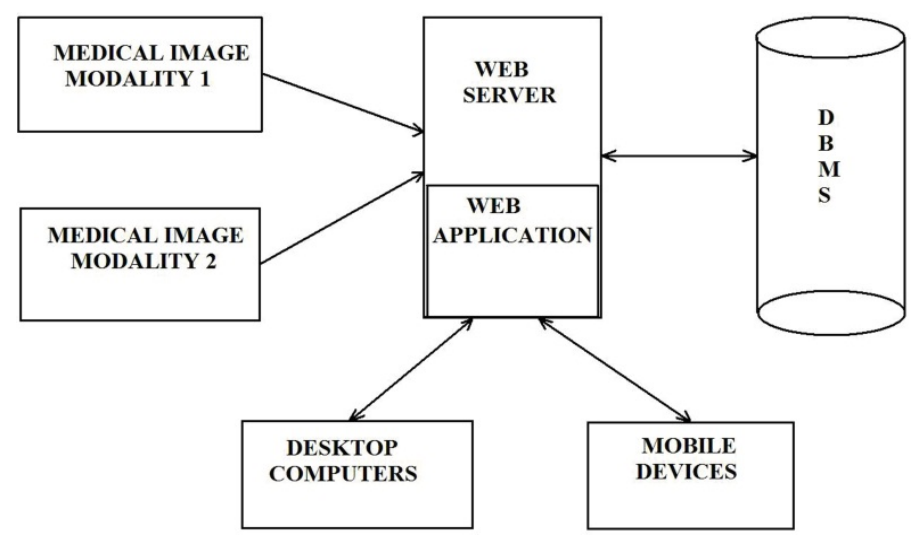

Figure 2. Web application architecture 
The possible states of the applications could be observed in the diagram shown in Figure 3 . The available tasks depend on the user type.

Figure 4 shows the list of actions that can be performed by a physician.

The proposed architecture and the developed components may be integrated in a ubiquitous computing system for dynamic adaptability [9].

For fusion purpose the selected images are displayed in a web page and the user can select the method to be applied. Figure 5 shows the web page for fusion action.

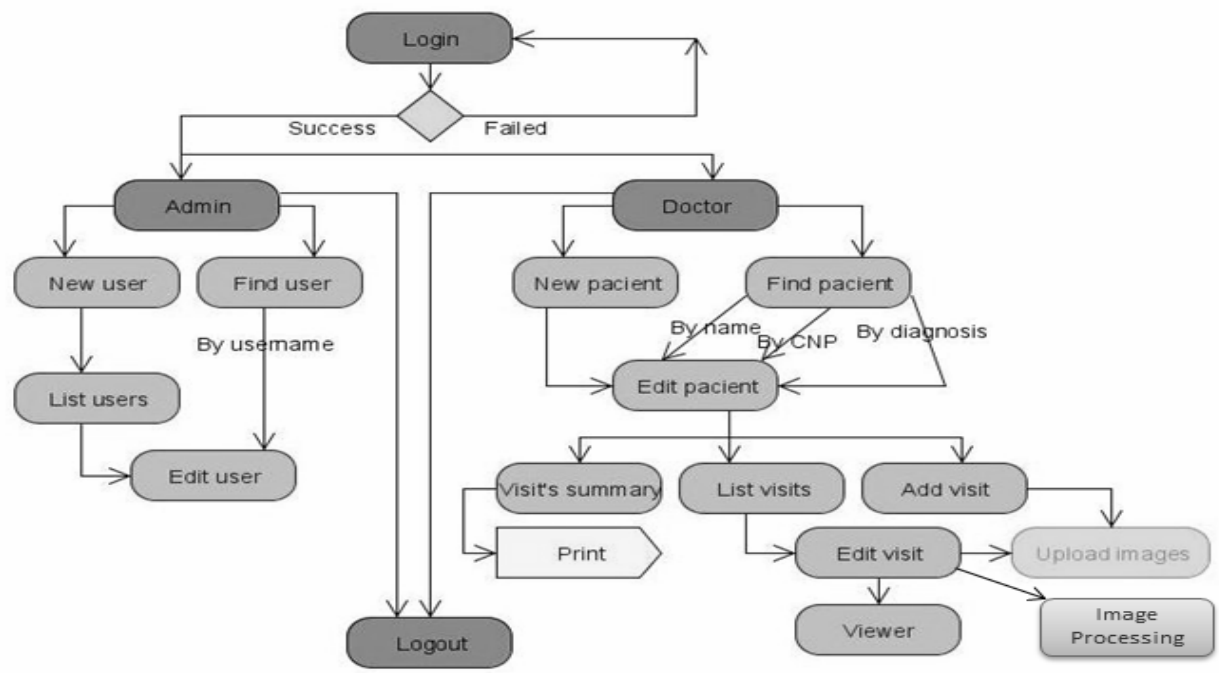

Figure 3. The state diagram of the application

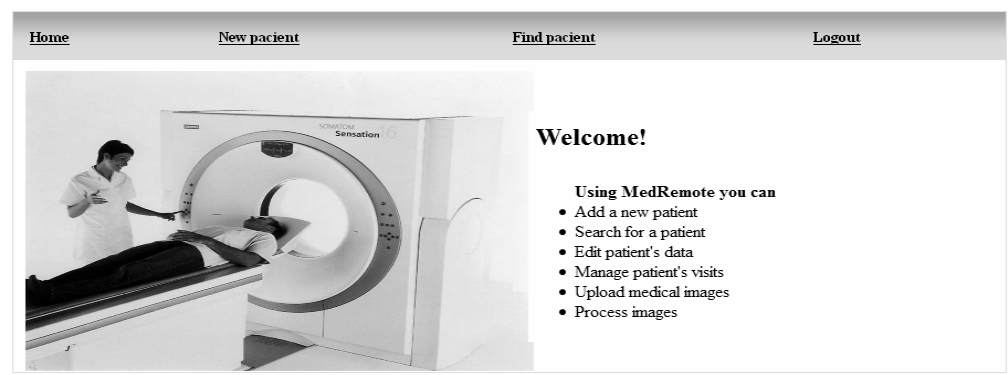

Figure 4. Welcome page for physician account

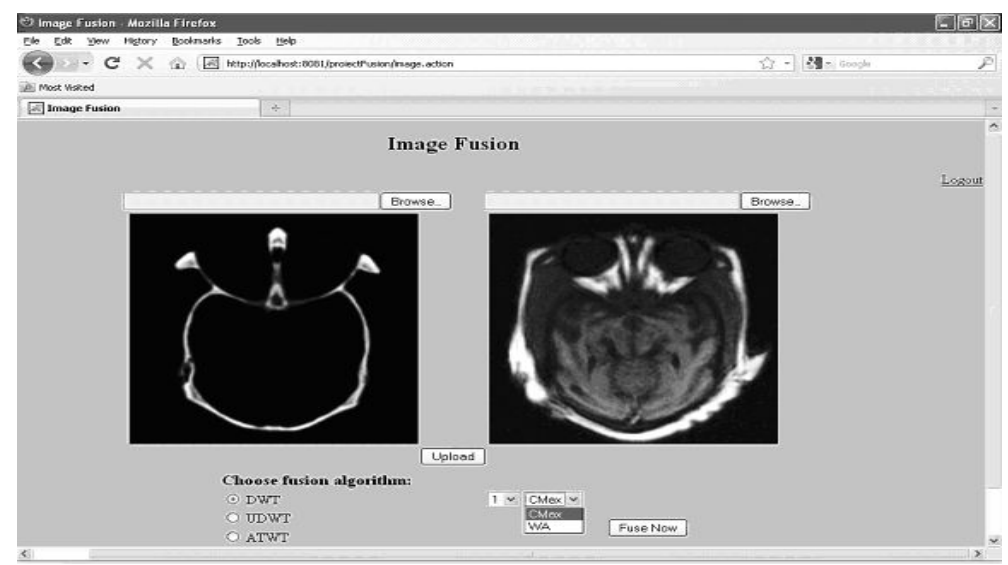

Figure 5. Selection and upload the images 


\section{Results}

In order to compare the implemented fusion techniques, a series of tests were carried out on different types of images. Each set of images (CT-MRI, MRI-SPECT and MRIPET) was used in the testing process of the fusion methods (DWT, UDWT and ATWT). The results are shown in Figure 6.

An objective comparison of the fusion methods may be realized based on the quality factors computed in the application. Tables 13 provide a quantitative assessment of the obtained fusion results.

Table 1 lists the results obtained for fusion factor and fusion symmetry for different radiological images, using CMax fusion rule.

Table 1. Results obtained for fusion factor and fusion symmetry

\begin{tabular}{|c|c|c|c|}
\hline $\begin{array}{c}\text { Image } \\
\text { type }\end{array}$ & $\begin{array}{c}\text { Multiresolution } \\
\text { decomposition } \\
\text { method }\end{array}$ & FF & FS \\
\hline MRI - & Haar DWT & 4.153 & 0.360 \\
\cline { 2 - 4 } CT & UDWT & 3.771 & 1.117 \\
\cline { 2 - 4 } & ATWT & 5.236 & 0.273 \\
\hline \multirow{2}{*}{$\begin{array}{c}\text { MRI }- \\
\text { PET }\end{array}$} & Haar DWT & 2.973 & 0.016 \\
\cline { 2 - 4 } & UDWT & 5.880 & 0.192 \\
\cline { 2 - 4 } & ATWT & 9.125 & 0.148 \\
\hline MRI - & Haar DWT & 1.867 & 0.010 \\
\cline { 2 - 4 } SPECT & UDWT & 3.940 & 0.086 \\
\cline { 2 - 4 } & ATWT & 27.099 & 0.018 \\
\hline
\end{tabular}

On the other hand, the fusion quality may be evaluated using the Piella's factors. The values obtained from tests of the above mentioned images are shown in Table 2.

Table 2. Results obtained for Piella's quality factors using CMax fusion rule

\begin{tabular}{|c|c|c|c|}
\hline $\begin{array}{c}\text { Image } \\
\text { type }\end{array}$ & $\begin{array}{c}\text { Multiresolution } \\
\text { decomposition } \\
\text { method }\end{array}$ & $\mathbf{Q}$ & Qw \\
\hline \multirow{2}{*}{$\begin{array}{c}\text { MRI- } \\
\text { CT }\end{array}$} & DWT & 0.6017 & 0.6493 \\
\cline { 2 - 4 } & UDWT & 0.3034 & 0.5853 \\
\hline \multirow{2}{*}{$\begin{array}{c}\text { MRI }- \\
\text { PET }\end{array}$} & ATWT & 0.5682 & 0.7383 \\
\cline { 2 - 4 } & UDWT & 0.4267 & 0.6651 \\
\hline \multirow{2}{*}{$\begin{array}{c}\text { MRI- } \\
\text { SPEC } \\
\text { T }\end{array}$} & ATWT & 0.3722 & 0.7144 \\
\cline { 2 - 4 } & DWT & 0.5496 & 0.8725 \\
\cline { 2 - 4 } & ATWT & 0.1749 & 0.3476 \\
\hline \multirow{2}{*}{ ATW } & 0.2490 & 0.6191 \\
\hline
\end{tabular}

$\mathrm{Q}$ - Piella fusion quality index

Qw - weighted quality index
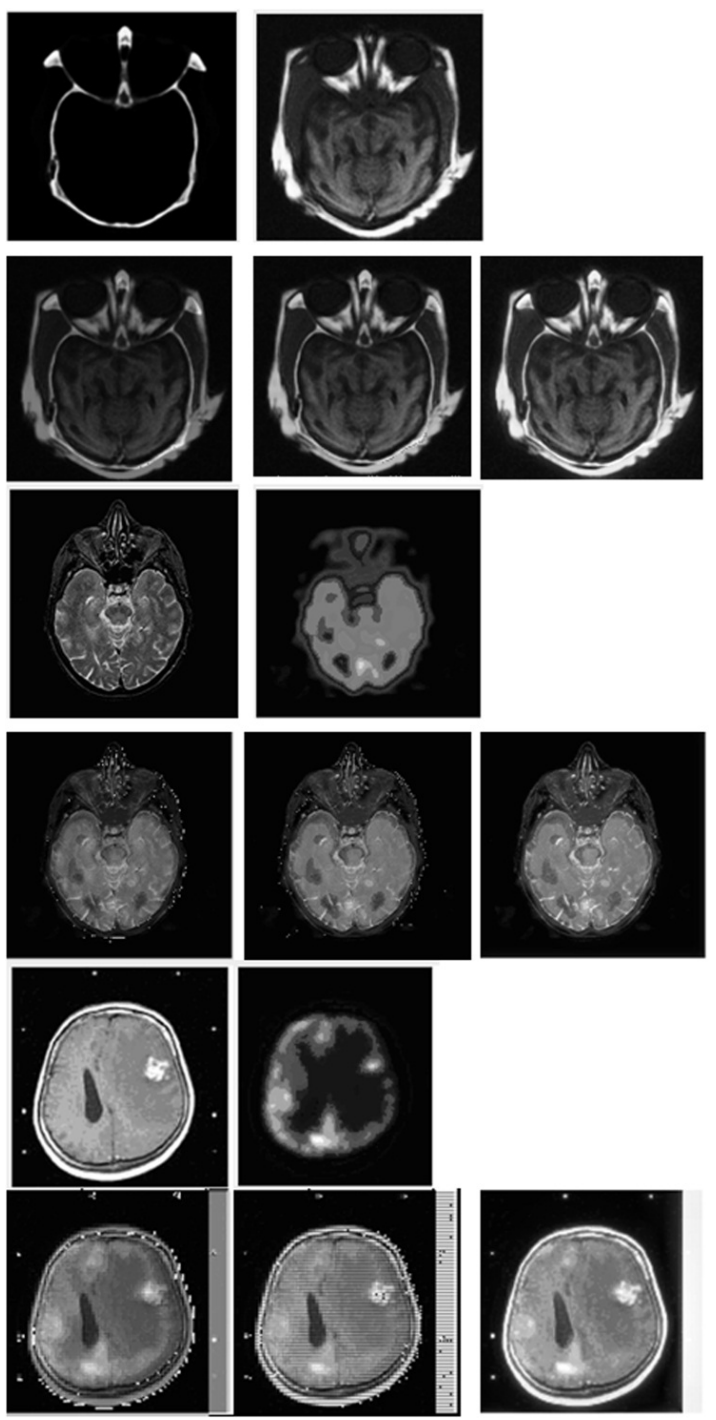

Figure 6. Original images (odd rows) and fusion results (even rows) between: CT and MRI, MRI and SPECT, MRI and PET images, using DWT Haar, UDWT and ATWT transforms and CMax fusion rule.

In order to compare the results obtained with different fusion rules, we offer the possibility of using CMax (choose-max) fusion rule or WA (weighted average) method proposed by Burt and Kolczinski. Some comparative results obtained with DWT Haar are listed in Table 3.

Table 3. Comparison between WA and CMax fusion rule

\begin{tabular}{|c|c|c|c|}
\hline $\begin{array}{c}\text { Image } \\
\text { type }\end{array}$ & $\begin{array}{c}\text { Fusion } \\
\text { rule }\end{array}$ & FF & FS \\
\hline CT-MRI & WA & 22.781 & 0.424 \\
\cline { 2 - 4 } & CMax & 4.153 & 0.360 \\
\hline MRI- & WA & 2.426 & 0.054 \\
\cline { 2 - 4 } SPECT & CMax & 1.867 & 0.010 \\
\hline \multirow{2}{*}{$\begin{array}{c}\text { MRI- } \\
\text { PET }\end{array}$} & WA & 5.263 & 0.386 \\
\cline { 2 - 4 } & CMax & 2.973 & 0.016 \\
\hline
\end{tabular}




\section{Conclusions}

In this paper we have proposed a software architecture that integrates image processing components able to be discovered in a ubiquitous computing system.

Our web distributed application offers the possibility of selecting fusion methods based on wavelet transform and fusion rules to emphasize different image features in the result image. A comparative presentation of the methods was made in the context of medical image fusion. These methods are implemented using Java technology, in packages that represent software components. Assisted diagnosis in healthcare may integrate such components. The fusion performance parameters offer a quantitative evaluation of the process being tested on different types of medical images. The results listed in Table 1 and Table 2 show the superiority of the ATWT over DWT, in decimated or undecimated form.

Future development of the application would consider providing software packages for image registration tasks. Assisted diagnosis based on registered images involved in the fusion process would be very useful in the healthcare domain.

\section{Acknowledgements}

This work was supported by CNCSIS UEFISCSU, project number PNII - IDEI $1083 / 2007-2010$ and co-financed from SIDOC - POSDRU/88/1.5/S/60078 project.

\section{REFERENCES}

1. AIAZZI, B., S. BARONTI, M. SELVA, Image Fusion Through Multiresolution Oversampled Decompositions, in $T$. Stathaki, Ed., Image fusion, Algorithms and applications, Elsevier Academic Press, UK, 2008, pp. 27-66.

2. BEYERER, J., M. HEIZMANN, J. SANDER, I. GHETA, Bayesian Methods for Image Fusion, in $T$. Stathaki, Ed., Image fusion, Algorithms and applications, Elsevier Academic Press, UK, 2008, pp. 157-192.
3. BURT, P. J., R. J. KOLCZYNSKI, Enhanced Image Capture through Fusion, Proceedings of the Fourth International Conference on Computer Vision, May 1993, Berlin, pp. 173-182.

4. GUIHONG, Q., Z. DALI, Y. PINGFAN, Medical Image Fusion by Wavelet Transform Modulus Maxima, Optics Express, vol.9, no. 4, 2001, pp. 184-190.

5. HILL, P., N. CANAGARAJAH, D. BULL, Image Fusion using Complex Wavelets, In Proceeding of the 13th British Machine Vision Conference BMVC 2002, September 2002, Cardiff, pp. 487-496.

6. NIKOLOV, S., P. HILL, D. BULL, N. CANAGARAJAH, Wavelets for Image Fusion, in S. Petrosian and F. Meyer, Ed., Wavelets in signal and image analysis, Computational Imaging and Vision Series, Kluwer Academic Publishers, Dordrecht, The Netherlands, 2001, pp. 213-244.

7. OTAZU, X., Theory and Implementation of Image Fusion Methods Based on the a Trous Algorithm, in T. Stathaki, Ed., Image fusion, Algorithms and applications, Elsevier Academic Press, UK, 2008, pp. 139-155.

8. PIElla, G., H. HEIJMANS, A New Quality Metric for Image Fusion, in Proceeding of International Conference on Image Processing, vol. 3, issue 2, September 2003, pp. 173-176.

9. POP, F. C., M. F. VAIDA, M. CREMENE, M. RIVEILL, C. PUIA, Dynamic Service Composition for Plug-and-Play Medical Devices, International Conference on Advancements of Medicine and Health Care through Technology, IFMBE Proceedings, Meditech09, vol. 26, September 2009, pp. 57-62.

10. ROCKINGER, O., Image Sequence Fusion Using a Shift Invariant Wavelet Transform, in Proceedings of the IEEE International Conference on Image Processing, vol.3, 1997, pp. 288-291.

11. SASIKALA, M., N. KUMARAVEL, A Comparative Analysis of Feature 
Based Image Fusion Methods, Information Technology Journal, Asian Network for Scientific Information, vol. 6, issue 8, 2007, pp. 1124-1230.

12. WANG, Q., Y. SHEN, J. JIN, Performance Evaluation of Image Fusion Techniques, in T. Stathaki, Ed., Image fusion, Algorithms and applications, Elsevier Academic Press, UK, 2008, pp. 469-492.
13. WANK, Z., A. C. BOVIK, H. R. SHEIKH, E. P. SIMONCELLI, Image Quality Assessment: From Error Visibility to Structural Similarity, In Proceeding of Transaction on Image Processing vol. 3, issue 4, April 2004, pp. 600-612. 